\title{
BRIEF REPORT \\ Acquired Monosomy 7 Myelodysplastic Syndrome in a Child With Clinical Features Suggestive of Dyskeratosis Congenita and IMAGe Association
}

\author{
Sharon McDonald, MD, ${ }^{1 *}$ David B. Wilson, MD, PhD, ${ }^{1,2}$ Elena Pumbo, $\mathrm{BS}^{3}{ }^{3}$ Shashikant Kulkarni, $\mathrm{PhD},{ }^{1}$ \\ Philip J. Mason, PhD, ${ }^{3,4}$ Tobias Else, $\mathrm{MD}^{5}$ Monica Bessler, $\mathrm{MD}, \mathrm{PhD},{ }^{2,3}$ Thomas Ferkol, $\mathrm{MD}{ }^{1}$ and Shalini Shenoy, $\mathrm{MD}^{1}$
}

We describe a case of acquired monosomy 7 myelodysplastic syndrome (MDS) in a boy with congenital adrenocortical insufficiency, genital anomalies, growth delay, skin hyperpigmentation, and chronic lung disease. Some of his clinical manifestations were suggestive of dyskeratosis congenita (DC), while other features resembled IMAGe association. DC has been linked to mutations in telomere maintenance genes. The genetic basis of IMAGe association is unknown, although mice harboring a mutation in a telomere maintenance gene, Tpp1, have adrenal hypoplasia congenita. We considered the possibility that this patient has a defect in telomere function resulting in features of both DC and IMAGe association. Pediatr Blood Cancer 2010;54:154-157. (c) 2009 Wiley-Liss, Inc.

Key words: adrenal cortex; dyskeratosis congenita; myelodysplasia; shelterin; telomere

\section{INTRODUCTION}

Monosomy 7 or partial loss of $7 q$ is found in $30 \%$ of children with myeloid disorders and is characterized by ineffective hematopoiesis, dysplastic bone marrow, and risk of evolution into acute leukemia [1]. Children with inherited bone marrow failure syndromes (IBMFS) are predisposed to myelodysplastic syndrome (MDS). One such IBMFS is dyskeratosis congenita (DC), a disorder that classically presents with bone marrow dysfunction and mucocutaneous features (abnormal pigmentation, dystrophic nails, and oral leukoplakia) [2]. Many patients with DC, however, lack the classic mucocutaneous features and have other somatic abnormalities such as lung disease, esophageal stricture, liver cirrhosis, osteoporosis, genitourinary anomalies, and cerebellar hypoplasia [2]. Mutations in six different genes (DKC1, NOP10, NHP2, TERC, TERT, and TINF2) cause DC, but account for only half of the patients diagnosed with this disorder [2,3]. Five of these genes (DKC1, NOP1O, NHP2, TERC, and TERT) encode components of the telomerase complex. The sixth gene mutated in DC, TINF2, encodes a protein component (TIN2) of the telomere ribonucleoprotein complex shelterin that safeguards telomeres [4]. The finding that all known genes affected in DC are involved in telomere maintenance suggests that telomere dysfunction plays a central role in the pathogenesis of DC and possibly other IBMFS [5,6].

We describe a male with acquired monosomy 7 MDS and manifestations of DC who underwent successful allogeneic hematopoietic stem cell transplantation (HSCT). Interestingly, this patient also has congenital adrenal insufficiency and other features of the rare, presumably autosomal recessive, endocrine disorder IMAGe association [intrauterine growth retardation (IUGR), metaphyseal dysplasia, adrenal hypoplasia congenita, genital anomalies; OMIM 300290] [7-13]. The genital anomalies of IMAGe association, which are restricted to males, include micropenis, hypospadias, cryptorchidism, and bifid scrotum [13]. Individuals with IMAGe association also have craniofacial anomalies (e.g., frontal bossing, broad nasal bridge, and low-set ears) [7-13]. Skin hyperpigmentation, reminiscent of that found in DC, has also been reported in IMAGe association [8]. The genetic basis of IMAGe association is unknown, although mice harboring a mutation in another telomere maintenance gene, Tpp1, have adrenal hypoplasia congenita and other hallmarks of this disorder $[14,15]$.
We considered the possibility that this patient may have defective telomere function resulting in features of both DC and IMAGe association.

\section{CASE REPORT}

The Caucasian male was born at 32 weeks gestational age to nonconsanguinous parents. The family history was unremarkable. The pregnancy was complicated by placenta previa and IUGR. Delivery was via caesarian section. APGAR scores were 7 at 1 min and 8 at $5 \mathrm{~min}$, and the birth weight was $1,157 \mathrm{~g}$. As a neonate, he was noted to have bifrontal bossing, broad nasal bridge, low-set ears (Fig. 1A), and genital anomalies (microphallus, hypospadias, and bilateral cryptorchidism). Chromosome analysis revealed a 46,XY genotype. He received testosterone replacement therapy with a good response in phallic and testicular enlargement and underwent orchiopexy to correct his cryptorchidism.

In the first week of life he developed hyponatremia and hyperkalemia. Endocrinologic evaluation revealed an elevated plasma adrenocorticotrophic hormone level with a low plasma cortisol level at less than $0.7 \mu \mathrm{g} / \mathrm{dl}$ (normal range $4-22 \mu \mathrm{g} / \mathrm{dl}$ ) and

${ }^{1}$ Department of Pediatrics, Washington University School of Medicine, St. Louis Children's Hospital, St. Louis, Missouri; ${ }^{2}$ Department of Developmental Biology, Washington University School of Medicine, St. Louis Children's Hospital, St. Louis, Missouri; ${ }^{3}$ Department of Internal Medicine, Washington University School of Medicine, St. Louis Children's Hospital, St. Louis, Missouri; ${ }^{4}$ Department of Genetics, Washington University School of Medicine, St. Louis Children's Hospital, St. Louis, Missouri; ${ }^{5}$ Department of Internal Medicine, University of Michigan, Ann Arbor, Michigan

The authors have no conflicts of interest to disclose.

Grant sponsor: NIH; Grant number: CA105312; Grant sponsor: St. Baldrick's Foundation; Grant sponsor: Hereditary Cancer Core of the Siteman Cancer Center; Grant sponsor: Tissue Procurement Core Siteman Cancer Center; Grant sponsor: Genome Sequencing Center Washington University St. Louis.

*Correspondence to: Sharon McDonald, Department of Pediatrics, Washington University School of Medicine, 660 S. Euclid Ave., Box 8116, St. Louis, MO 63110. E-mail: mcdonald_s@wustl.edu

Received 23 June 2009; Accepted 12 August 2009 

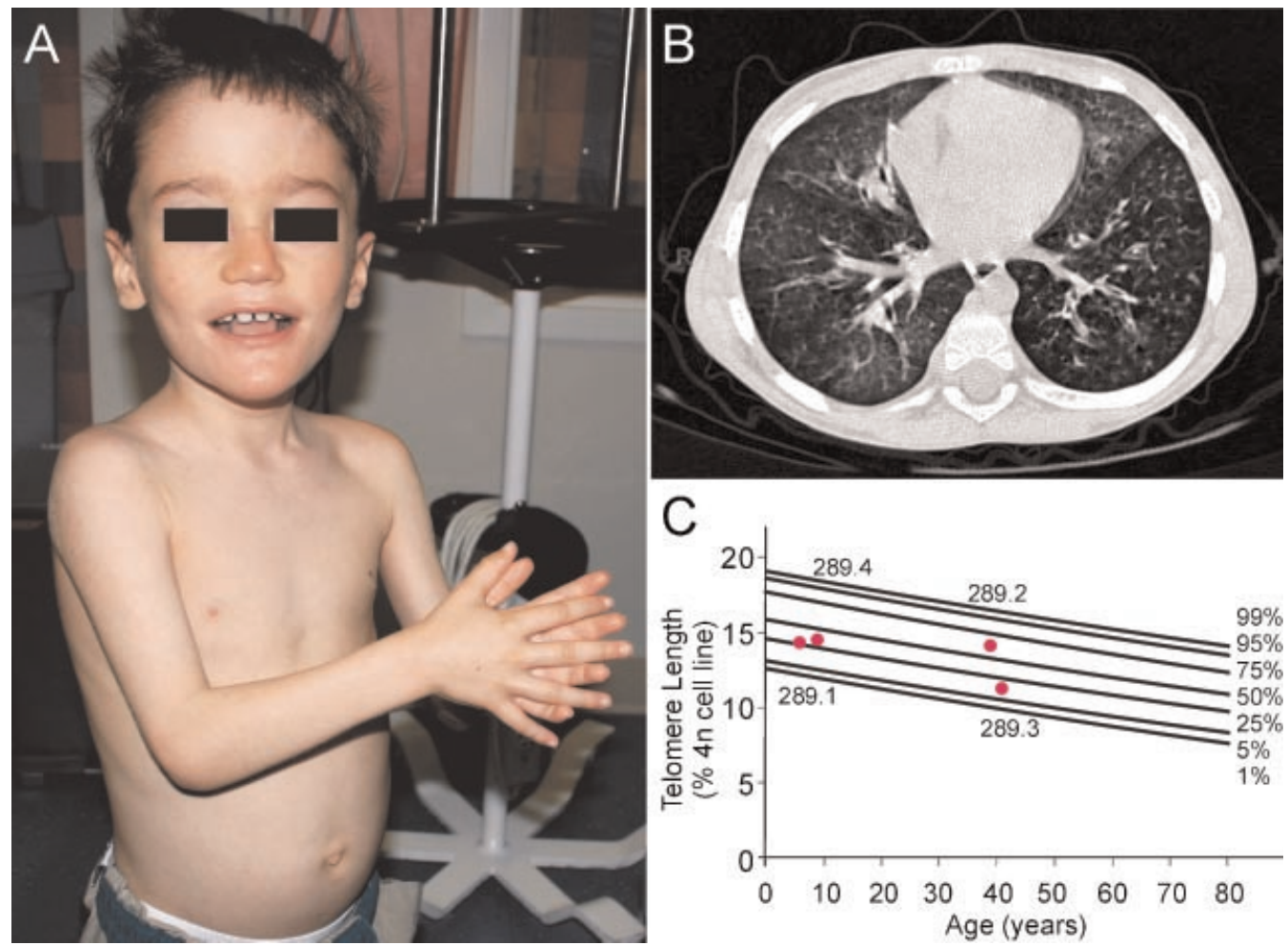

Fig. 1. A: Picture of the patient at 5 years of age. Note the disproportionately large head, prominent forehead, broad nasal bridge, low-set ears, and thin arms. The physical appearance of our patient resembles that of individuals with IMAGe association [10]. B: Computed tomography of the chest at 4 years of age showing extensive pulmonary disease with bronchiectasis and tree-in-bud infiltrates bilaterally. C: PBMC telomere length measurements on the proband and his unaffected first degree relatives. Lines represent percentiles (1-99\%) of telomere length measured in 234 healthy individuals between the ages of 0.3 and 94 years old. Red circles indicate the telomere lengths for proband and his relatives. The proband is indicated as 289.1, mother as 289.2, father as 289.3 , and sibling as 289.4. All family members had normal telomere lengths. [Color figure can be viewed in the online issue, which is available at www.interscience.wiley.com.]

normal plasma 17-hydroxyprogesterone level at 40 (normal range 26-568) resulting in the diagnosis of adrenal hypoplasia congenita. A co-syntropin stimulation test at 20 days of age revealed a baseline cortisol level of $<0.7 \mu \mathrm{g} / \mathrm{dl}$ and a stimulation level of $3.2 \mu \mathrm{g} / \mathrm{dl}$, supporting the diagnosis of primary adrenocortical insufficiency.

As a toddler he had feeding difficulties due to uncoordinated swallowing and esophageal stricture. He underwent esophagoscopy with dilation at 2 and 4 years of age. Esophageal biopsy showed ballooning degeneration of the squamous epithelium. Despite adequate caloric intake, his weight and length remained $<3$ rd percentile throughout childhood. At the chronologic age of 4.3 years, his bone age was 3.7 years. MRI of his brain at 16 months of age showed a large extracerebral cerebrospinal fluid space in his left frontal region and multiple diffuse periventricular white matter lesions with abnormal myelination. Extensive genetic testing was performed on the patient, and the results are summarized in Table I.

As an infant and toddler he had numerous hospitalizations for recurrent pneumonia. Chest radiographs and computed tomography of the chest showed diffuse bilateral ground glass opacities superimposed on bronchiectasis and bronchiolectasis (Fig. 1B). Bronchoscopy revealed a normal tracheal lining, increased mucosal vascularity, and thick, clear secretions throughout the bronchial tree. Biopsy of the respiratory epithelium demonstrated normal cilia on electron microscopy. In addition to lung infections, he had frequent episodes of otitis media with associated hearing loss, necessitating myringotomies with tympanostomy tube placement. An immuno- deficiency workup revealed a low Haemophilus influenzae titer and low $\mathrm{IgG}$ for which he received intravenous immunoglobulin replacement therapy.

At 3 years of age, he developed persistent neutropenia. Bone marrow evaluation revealed myeloid hyperplasia with atypia, neutrophil hypolobation, and hypergranulation. Cytogenetic analysis revealed monosomy 7 in 18 of 20 metaphases. FISH analysis of buccal mucosal cells and fibroblasts revealed no monosomy 7 . With written informed consent of the parents and approval of the Institutional Review Board, peripheral blood mononuclear cell (PBMC) telomere length measurements [16] were performed on the patient and his family (Fig. 1C). There was no telomere shortening present in either the patient or his parents. A series of telomere maintenance genes were also sequenced (TERC, TERT, TINF2, $T P P 1$, and $D K C 1$ ), but no mutations were detected.

At 6 years of age, he underwent allogenic HSCT from a 10/10 allele matched unrelated donor. The preparative regimen included fludarabine $120 \mathrm{mg} / \mathrm{m}^{2}$ and melphalan $140 \mathrm{mg} / \mathrm{m}^{2}$ [17]. Graftversus-host disease (GVHD) prophylaxis included methotrexate, cyclosporine A, and prednisone. He tolerated HSCT without acute complications. A skin rash developed 8 months after transplant and was consistent with GVHD restricted to the skin. Topical hydrocortisone induced minimal improvement but therapeutic levels of cyclosporine $(250-350 \mathrm{ng} / \mathrm{ml})$ induced remission with residual localized hyperpigmentation. Mucus membranes and other organs remained unaffected and cyclosporine was weaned again 
TABLE I. Genetic Disease Evaluations Performed

\begin{tabular}{llr}
\hline Test & \multicolumn{1}{c}{ Associated disorder } & Result \\
\hline Sweat chloride & Cystic fibrosis & Normal \\
CFTR sequence & Cystic fibrosis & Normal \\
Alpha-1-antitrypsin & Alpha-1-antitrypsin deficiency & Normal \\
7-Dehydrocholesterol level & Smith-Lemli-Opitz syndrome & Normal \\
Xp21.3 microdeletion & Glycerol kinase deficiency & Normal \\
DAX1 (NROB1) sequence & Congenital adrenal hypoplasia & Normal \\
SF1 $(N R 5 A 1)$ sequence & Gonadal dysgenesis and adrenal failure & Normal \\
Aprataxin sequence & Ataxia-oculomotor apraxia & Normal \\
Nibrin $(N B S 1)$ level & Nijmegen breakage syndrome & Normal \\
Mre11 (ATLD) level & Ataxia-telangiectasia & Normal \\
ATM gene sequence and radiosensitivity testing & Ataxia-telangiectasia & Normal \\
Diepoxybutane chromosome fragility & Fanconi anemia & Normal \\
$T E R C$ sequence & Dyskeratosis congenita & Normal \\
$T E R T$ sequence & Dyskeratosis congenita & Normal \\
$T I N F 2$ sequence & Dyskeratosis congenita & Normal \\
$T P P 1(A C D)$ sequence & Dyskeratosis congenita & Normal \\
$D K C 1$ sequence & Dyskeratosis congenita & Normal \\
Adenosine deaminase & Severe combined immunodeficiency & \\
Purine nucleoside phosphorylase & Severe combined immunodeficiency & \\
\hline
\end{tabular}

CFTR, cystic fibrosis transmembrane conductance regulator; DAX1, dosage sensitive sex reversal, adrenal hypoplasia critical region, on chromosome X, gene 1; SF1, steroidogenic factor 1; ATM, ataxia-telangiectasia mutated; TERC, telomerase RNA component; TERT, telomerase reverse transcriptase; TINF2, TERF-interacting nuclear factor 2; TPP1, TIN2-interacting protein 1; DKC1, dykserin; ACD, adrenocortical dysplasia.

gradually. Complete donor engraftment with no evidence of monosomy 7 was documented at 1 year.

In the post-transplant period, he developed several lower respiratory infections, likely secondary to immunosuppression and underlying bronchiectasis. Accordingly, he was maintained on alternating courses of levofloxacin and ciprofloxacin prophylaxis until 1 year post-transplant, and monthly infusions of intravenous immunoglobulin therapy $(400 \mathrm{mg} / \mathrm{kg} / \mathrm{dose})$ for 15 months.

\section{DISCUSSION}

Although he lacked the classic mucocutaneous features of DC, the proband had other hallmarks of DC including IUGR, MDS, esophageal stricture, lung disease, and hypospadias. DC is a telomere maintenance disorder and is often, but not always, associated with very short telomeres [16]. Although the PBMC telomere length was normal, this was measured after the diagnosis of MDS, and the surviving MDS hematopoietic cells may not have reflected the average telomere length in other tissues. For this reason telomere length measurements must be interpreted with caution [16]. He did not harbor a mutation in TERC, TERT, DKC1, or TINF2, but only about half of patients with DC have an identifiable mutation in these telomere maintenance genes.

A cardinal finding in our patient, congenital adrenal insufficiency, has not been linked to DC in humans. However, mice harboring a hypomorphic allele of Tpp1, a component of the shelterin complex that protects telomeres, exhibit a complex phenotype that includes adrenocortical hypoplasia with cytomegaly [14,15]. This allele, termed Tpp1 acd (for the adrenocortical dysplasia strain), results in telomeres that are dysfunctional, leading to an increase in senescence-associated markers in adrenocortical cells. These findings suggest that Tppl is essential for proper function of progenitor cells in the adrenal cortex and that abnormalities in telomere maintenance can, at least in mice, manifest as adrenocort- ical dysfunction. Investigators are currently searching for mutations in telomere maintenance genes in humans with certain forms of congenital adrenocortical insufficiency $[7,18,19]$. We did not detect a TPP1 mutation in our patient.

One other patient with monosomy 7 syndrome, congenital adrenal hypoplasia with cytomegaly, and male pseudohermaphroditism has been previously described by Le and Kutteh [20]. We speculate that our patient and that of Le and Kutteh have a still undefined defect in telomere function with features of both DC and IMAGe association. Clinically well-delineated cases such as these may help elucidate the molecular pathogenesis of MDS and telomere maintenance disorders. While the hematopoietic manifestations of MDS and monosomy 7 were successfully tackled in our patient with reduced intensity allogeneic HSCT, other systemic manifestations such as adrenal and pulmonary complications continue to require ongoing management.

\section{ACKNOWLEDGMENT}

The authors thank the patient and his family for their participation. The work was supported in part by the NIH grant "Molecular Studies on BMF" (http://bmf.im.wustl.edu) CA105312 (to M.B., P.J.M., and D.B.W.) as well as the St. Baldrick's Foundation (to S.M.). We are grateful to the Hereditary Cancer Core of the Siteman Cancer Center for patent enrollment, Tissue Procurement Core Siteman Cancer Center for DNA extraction and sample storage, and the Genome Sequencing Center Washington University St. Louis for mutation screening.

\section{REFERENCES}

1. Aktas D, Tuncbilek E. Myelodysplastic syndrome associated with monosomy 7 in childhood: A retrospective study. Cancer Genet Cytogenet 2006;171:72-75. 
2. Bessler M, Du HY, Gu B, et al. Dysfunctional telomeres and dyskeratosis congenita. Haematologica 2007;92:1009-1012.

3. Vulliamy T, Dokal I. Dyskeratosis congenita. Semin Hematol 2006;43:157-166.

4. Savage SA, Giri N, Baerlocher GM, et al. TINF2, a component of the shelterin telomere protection complex, is mutated in dyskeratosis congenita. Am J Hum Genet 2008;82:501-509.

5. Savage SA, Alter BP. The role of telomere biology in bone marrow failure and other disorders. Mech Ageing Dev 2008;129:35-47.

6. Calado RT, Young NS. Telomere maintenance and human bone marrow failure. Blood 2008;111:4446-4455.

7. Hutz JE, Krause AS, Achermann JC, et al. IMAGe association and congenital adrenal hypoplasia: No disease-causing mutations found in the ACD gene. Mol Genet Metab 2006;88:66-70.

8. Ko JM, Lee JH, Kim GH, et al. A case of a Korean newborn with IMAGe association presenting with hyperpigmented skin at birth. Eur J Pediatr 2007;166:879-880.

9. Lienhardt A, Mas JC, Kalifa G, et al. IMAGe association: Additional clinical features and evidence for recessive autosomal inheritance. Horm Res 2002;57(Suppl 2):71-78.

10. Pedreira CC, Savarirayan R, Zacharin MR. IMAGe syndrome: A complex disorder affecting growth, adrenal and gonadal function, and skeletal development. J Pediatr 2004;144:274-277.

11. Tan TY, Jameson JL, Campbell PE, et al. Two sisters with IMAGe syndrome: Cytomegalic adrenal histopathology, support for autosomal recessive inheritance and literature review. Am J Med Genet A 2006;140:1778-1784.

12. Vilain E, Le MM, Lecointre C, et al. IMAGe, a new clinical association of intrauterine growth retardation, metaphyseal dysplasia, adrenal hypoplasia congenita, and genital anomalies. J Clin Endocrinol Metab 1999;84:4335-4340.

13. Bergadá I, Del RG, Lapunzina P, et al. Familial occurrence of the IMAGe association: Additional clinical variants and a proposed mode of inheritance. J Clin Endocrinol Metab 2005;90:31863190.

14. Else T, Theisen BK, Wu Y, et al. Tpp1/Acd maintains genomic stability through a complex role in telomere protection. Chromosome Res 2007;15:1001-1013.

15. Keegan CE, Hutz JE, Else T, et al. Urogenital and caudal dysgenesis in adrenocortical dysplasia (acd) mice is caused by a splicing mutation in a novel telomeric regulator. Hum Mol Genet 2005; 14 : 113-123.

16. Du HY, Pumbo E, Ivanovich J, et al. TERC and TERT gene mutations in patients with bone marrow failure and the significance of telomere length measurements. Blood 2009;113:309-316.

17. Shenoy S, Grossman WJ, DiPersio J, et al. A novel reducedintensity stem cell transplant regimen for nonmalignant disorders. Bone Marrow Transplant 2005;35:345-352.

18. Keegan CE, Hutz JE, Krause AS, et al. Novel polymorphisms and lack of mutations in the ACD gene in patients with ACTH resistance syndromes. Clin Endocrinol (Oxf) 2007;67:168174.

19. Else T. Telomeres and telomerase in adrenocortical tissue maintenance, carcinogenesis, and aging. J Mol Endocrinol 2009; doe:10.1677/JME-0800189 [in press].

20. Le SQ, Kutteh WH. Monosomy 7 syndrome associated with congenital adrenal hypoplasia and male pseudohermaphroditism. Obstet Gynecol 1996;87:854-856. 